Supplementary Table S1. Multivariable association of a 1-SD increase in FG-SD with CAC progression over 10 years among individuals in the CARDIA study

\begin{tabular}{|c|c|c|}
\hline Model & $\begin{array}{c}\text { \% CAC progression } \\
\text { per 1-SD increase in FG-SD }(95 \% \mathrm{CI})\end{array}$ & $P$ value \\
\hline \multicolumn{3}{|c|}{ Incident $\mathrm{CAC}^{*}$ ( $>0$ Agatston units), $\mathrm{N}=2,062$} \\
\hline Model 1 & $11.1(8.2,14.0)$ & $<0.001$ \\
\hline Model 2 & $10.6(7.8,13.4)$ & $<0.001$ \\
\hline Model 3 & $8.1(5.4,10.9)$ & $<0.001$ \\
\hline Model 4 & $8.6(4.9,12.2)$ & 0.019 \\
\hline Model 4A & $8.0(4.3,11.6)$ & 0.030 \\
\hline Model 4B & $8.6(5.0,12.3)$ & 0.018 \\
\hline \multicolumn{3}{|c|}{ Any CAC progression ${ }^{\dagger}$ ( $>0$ Agatston units), $N=2,256$} \\
\hline Model 1 & $10.9(8.3,13.5)$ & $<0.001$ \\
\hline Model 2 & $10.5(7.9,13.0)$ & $<0.001$ \\
\hline Model 3 & $7.7(5.3,10.1)$ & $<0.001$ \\
\hline Model 4 & $9.5(6.2,12.9)$ & 0.005 \\
\hline Model 4A & $8.9(5.6,12.3)$ & 0.008 \\
\hline Model 4B & $9.8(6.4,13.1)$ & 0.004 \\
\hline Model 4C & $9.2(5.9,12.5)$ & 0.006 \\
\hline
\end{tabular}

A 1-SD unit increment in FG-SD is $15.7 \mathrm{mg} / \mathrm{dL}$. Model 1, unadjusted; Model 2, adjusted for age, race and sex; Model 3, adjusted for variables in model 2 plus BMI, current smoker status (yes/no), milliliter of daily alcohol consumption, SBP, antihypertensive medication use (yes/no), physical activity, serum creatinine, total cholesterol and LDL-C; Model 4, adjusted for variables in model 3 plus average of FG and change in FG level during variability measurement; Model 4A, adjusted for variables in model 4 plus incidence of diabetes and diabetes medication use; Model 4B, adjusted for variables in model 4 plus FG level at year 25. Model 4C, for individuals with any CAC progression, adjusted for variables in model 4 plus baseline CAC. ${ }^{*}$ Evaluated only among individuals without baseline CAC at the year 15 examination. ${ }^{\dagger} \mathrm{CAC}$ progression was calculated as the difference of logarithmic CAC score at follow-up and baseline ( $\log$ [CAC (follow-up) +1$]-\log$ [CAC (baseline) + 1]). FG-SD, the standard deviation of fasting glucose. 
Supplementary Table S2. Multivariable association of a 1-SD increase in FG-ARV with CAC progression over 10 years among individuals in the CARDIA study

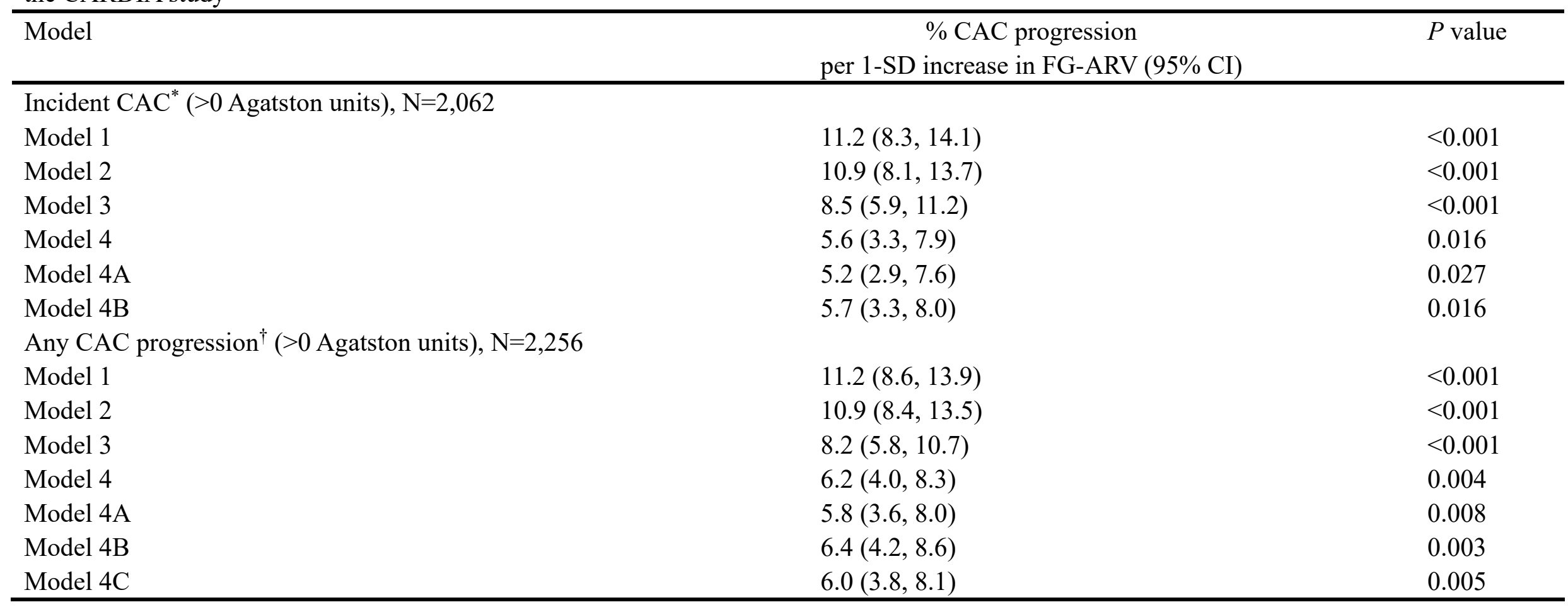

A 1-SD unit increment in FG-ARV is $19.2 \mathrm{mg} / \mathrm{dL}$ per year. Model 1, unadjusted; Model 2, adjusted for age, race and sex; Model 3, adjusted for variables in model 2 plus BMI, current smoker status (yes/no), milliliter of daily alcohol consumption, SBP, antihypertensive medication use (yes/no), physical activity, serum creatinine, total cholesterol and LDL-C; Model 4, adjusted for variables in model 3 plus average of FG and change in FG level during variability measurement; Model 4A, adjusted for variables in model 4 plus incidence of diabetes and diabetes medication use; Model 4B, adjusted for variables in model 4 plus FG level at year 25. Model 4C, for individuals with any CAC progression, adjusted for variables in model 4 plus baseline CAC. *Evaluated only among individuals without baseline CAC at the year 15 examination. ${ }^{\dagger} \mathrm{CAC}$ progression was calculated as the difference of logarithmic CAC score at follow-up and baseline (log [CAC (follow-up) +1$]-\log$ [CAC (baseline) + 1]). FG-ARV, the average real variability of fasting glucose. 
Supplementary Table S3. Multivariable association between FG-CV during young adulthood and CAC progression in middle age: CAC progression estimated by CAC volume score

\begin{tabular}{|c|c|c|}
\hline Model & $\begin{array}{c}\text { \% CAC progression } \\
\text { per 1-SD increase in } \mathrm{FG}-\mathrm{CV}(95 \% \mathrm{CI})\end{array}$ & $P$ value \\
\hline \multicolumn{3}{|c|}{ Incident $\mathrm{CAC}^{*}$ ( $>0$ volume score), $\mathrm{N}=2,023$} \\
\hline Model 1 & $8.3(5.6,11.1)$ & $<0.001$ \\
\hline Model 2 & $8.1(5.4,10.8)$ & $<0.001$ \\
\hline Model 3 & $5.9(3.3,8.5)$ & $<0.001$ \\
\hline Model 4 & $6.0(1.0,11.1)$ & 0.016 \\
\hline \multicolumn{3}{|c|}{ Any CAC progression $^{\dagger}$ ( $>0$ volume score), $\mathrm{N}=2,256$} \\
\hline Model 1 & $8.1(5.7,10.5)$ & $<0.001$ \\
\hline Model 2 & $7.9(5.6,10.3)$ & $<0.001$ \\
\hline Model 3 & $5.6(3.3,7.9)$ & $<0.001$ \\
\hline Model 4 & $6.6(2.7,10.5)$ & 0.003 \\
\hline
\end{tabular}

A 1-SD unit increment in FG-CV is 9.3\%. Model 1, unadjusted; Model 2, adjusted for age, race and sex; Model 3, adjusted for variables in model 2 plus BMI, current smoker status (yes/no), milliliter of daily alcohol consumption, SBP, antihypertensive medication use (yes/no), physical activity, serum creatinine, total cholesterol and LDL-C; Model 4, adjusted for variables in model 3 plus average of FG and change in FG level during variability measurement; Model 4A, adjusted for variables in model 4 plus incidence of diabetes and diabetes medication use; Model 4B, adjusted for variables in model 4 plus FG level at year 25. Model 4C, for individuals with any CAC progression, adjusted for variables in model 4 plus baseline CAC. ${ }^{*}$ Evaluated only among individuals without baseline CAC at the year 15 examination. ${ }^{\dagger} \mathrm{CAC}$ progression was calculated as the difference of logarithmic CAC volume score at follow-up and baseline $(\log$ [CAC (follow-up) +1$]-\log [\mathrm{CAC}$ (baseline) +1$]$ ). 
Supplementary Table S4. Multivariable association between FG-SD during young adulthood and CAC progression in middle age: CAC progression estimated by CAC volume score

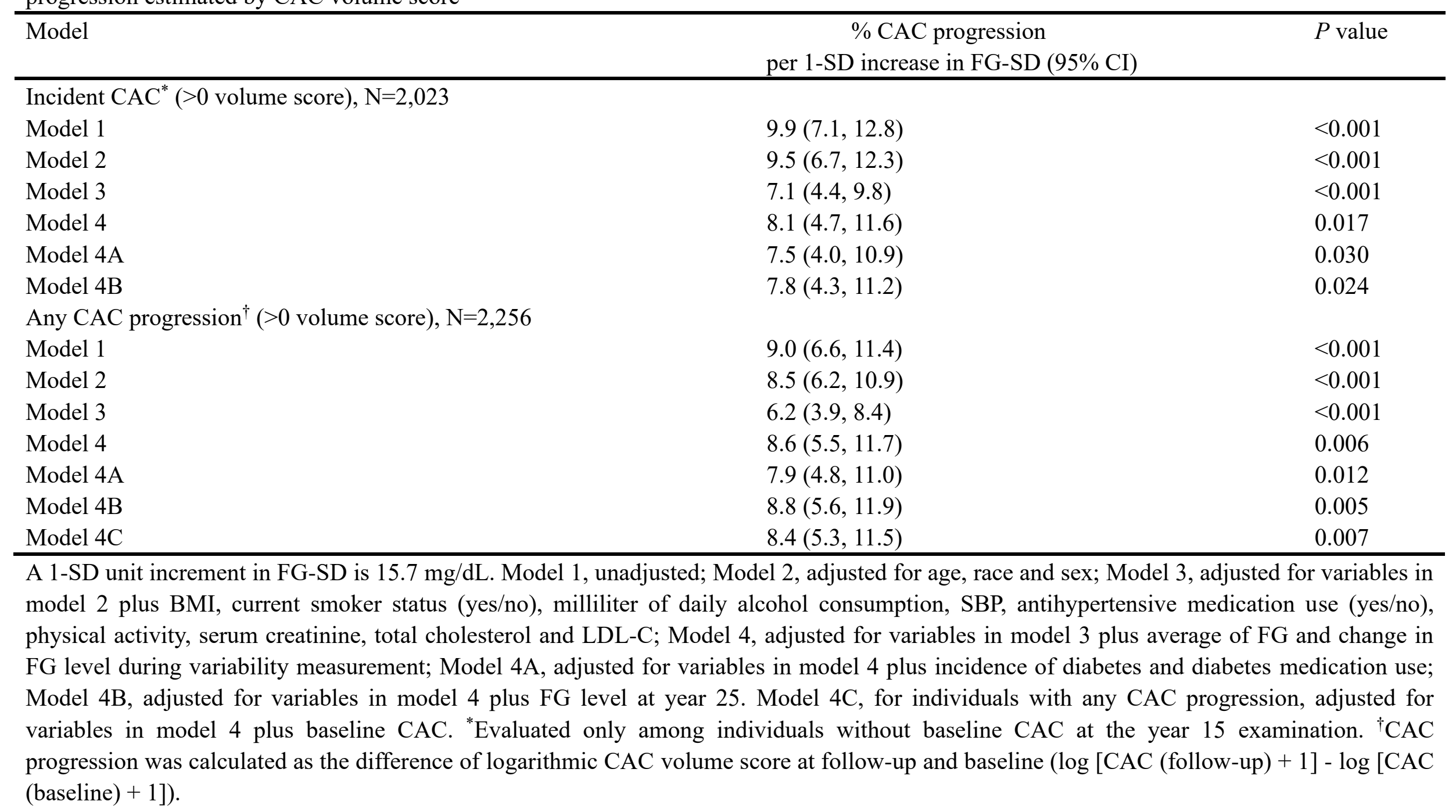


Supplementary Table S5. Multivariable association between FG-ARV during young adulthood and CAC progression in middle age: CAC progression estimated by CAC volume score

\begin{tabular}{|c|c|c|}
\hline Model & $\begin{array}{c}\% \text { CAC progression } \\
\text { per 1-SD increase in FG-ARV }(95 \% \mathrm{CI})\end{array}$ & $P$ value \\
\hline \multicolumn{3}{|c|}{ Incident $\mathrm{CAC}^{*}$ ( $>0$ Agatston units), $\mathrm{N}=2,023$} \\
\hline Model 1 & $9.8(7.0,12.6)$ & $<0.001$ \\
\hline Model 3 & $7.4(6.0,8.8)$ & $<0.001$ \\
\hline Model 4 & $5.2(3.0,7.4)$ & 0.017 \\
\hline \multicolumn{3}{|c|}{ Any CAC progression $^{\dagger}$ ( $>0$ Agatston units), $N=2,256$} \\
\hline Model 1 & $9.3(6.9,11.7)$ & $<0.001$ \\
\hline Model 2 & $9.0(6.6,11.3)$ & $<0.001$ \\
\hline Model 3 & $6.7(4.3,9.0)$ & $<0.001$ \\
\hline Model 4 & $5.4(3.4,7.4)$ & 0.008 \\
\hline Model 4A & $4.9(2.9,6.9)$ & 0.015 \\
\hline
\end{tabular}

A 1-SD unit increment in FG-ARV is $19.2 \mathrm{mg} / \mathrm{dL}$ per year. Model 1, unadjusted; Model 2, adjusted for age, race and sex; Model 3, adjusted for variables in model 2 plus BMI, current smoker status (yes/no), milliliter of daily alcohol consumption, SBP, antihypertensive medication use (yes/no), physical activity, serum creatinine, total cholesterol and LDL-C; Model 4, adjusted for variables in model 3 plus average of FG and change in FG level during variability measurement; Model 4A, adjusted for variables in model 4 plus incidence of diabetes and diabetes medication use; Model 4B, adjusted for variables in model 4 plus FG level at year 25. Model 4C, for individuals with any CAC progression, adjusted for variables in model 4 plus baseline CAC. *Evaluated only among individuals without baseline CAC at the year 15 examination. ${ }^{\dagger}$ CAC progression was calculated as the difference of logarithmic CAC volume score at follow-up and baseline (log [CAC (follow-up) +1$]-\log$ [CAC (baseline) + 1]). 
Supplementary Table S6. Multivariable association between fasting glucose variation during study participants with CAC progression over 10 years (2000-2010) stratified by diabetes status

\begin{tabular}{|c|c|c|c|c|c|c|}
\hline & Model 1 & Model 2 & Model 3 & Model 4 & Model 5 & Model 6 \\
\hline \multicolumn{7}{|c|}{ \% CAC progression per 1-SD increase in FG-CV $(95 \% \mathrm{CI})$} \\
\hline \multicolumn{7}{|l|}{ Incident $\mathrm{CAC}^{*}$} \\
\hline Diabetes, $n=129$ & $10.8(2.7,18.8)$ & $7.3(-0.3,14.9)$ & $6.8(-0.8,14.4)$ & $3.4(-9.6,16.3)$ & $-2.7(-14.0,8.7)$ & \\
\hline No Diabetes, $n=1,933$ & $6.7(3.4,10.0)$ & $6.9(3.7,10.2)$ & $4.8(1.6,7.9)$ & $6.4(0.7,12.1)$ & $3.4(-0.1,6.8)$ & \\
\hline \multicolumn{7}{|l|}{$P$ for interaction $=0.268$} \\
\hline \multicolumn{7}{|l|}{ Any CAC progression ${ }^{\dagger}$} \\
\hline Diabetes, $n=148$ & $9.5(2.3,16.6)$ & $7.6(0.9,14.2)$ & $6.7(0.0,13.4)$ & $2.0(-9.6,13.5)$ & $-2.3(-11.8,7.2)$ & $2.0(-9.5,13.5)$ \\
\hline No Diabetes, $n=2,108$ & $7.9(4.8,11.0)$ & $8.2(5.2,11.3)$ & $5.8(2.8,8.7)$ & $7.6(2.5,12.8)$ & $4.4(0.7,8.0)$ & $7.9(2.9,12.9)$ \\
\hline \multicolumn{7}{|l|}{$P$ for interaction $=0.642$} \\
\hline \multicolumn{7}{|c|}{$\%$ CAC progression per 1-SD increase in FG-SD (95\% CI) } \\
\hline \multicolumn{7}{|l|}{ Incident $\mathrm{CAC}^{*}$} \\
\hline Diabetes, $n=129$ & $11.1(3.8,18.4)$ & $7.9(1.0,14.9)$ & $6.8(-0.1,13.8)$ & $2.9(-14.8,20.7)$ & $-4.4(-17.8,9.0)$ & \\
\hline No Diabetes, $n=1,933$ & $9.0(5.5,12.5)$ & $8.9(5.5,12.4)$ & $6.5(3.3,9.6)$ & $9.7(5.6,13.8)$ & $4.7(2.0,7.4)$ & \\
\hline \multicolumn{7}{|l|}{$\mathrm{P}$ for interaction $=0.552$} \\
\hline \multicolumn{7}{|l|}{ Any CAC progression ${ }^{\dagger}$} \\
\hline Diabetes, $n=148$ & $10.4(3.8,16.9)$ & $8.6(2.5,14.7)$ & $7.4(1.2,13.6)$ & $1.4(-14.1,17.0)$ & $-3.7(-15.2,7.9)$ & $1.3(-14.2,16.8)$ \\
\hline No Diabetes, $n=2,108$ & $9.5(6.4,12.7)$ & $9.5(6.4,12.5)$ & $6.8(3.8,9.8)$ & $11.6(7.7,15.4)$ & $5.6(1.0,10.2)$ & $11.0(7.2,14.8)$ \\
\hline \multicolumn{7}{|l|}{$P$ for interaction $=0.798$} \\
\hline \multicolumn{7}{|c|}{$\%$ CAC progression per 1-SD increase in FG-ARV (95\% CI) } \\
\hline \multicolumn{7}{|c|}{ Incident $\mathrm{CAC}^{*}$} \\
\hline Diabetes, $n=129$ & $11.6(3.8,19.5)$ & $9.2(1.8,16.5)$ & $8.1(0.7,15.5)$ & $2.4(-9.4,14.2)$ & $0.4(-11.3,12.1)$ & \\
\hline No Diabetes, $n=1,933$ & $9.2(5.8,12.5)$ & $9.2(5.9,12.5)$ & $6.9(3.7,10.2)$ & $6.2(3.6,8.8)$ & $4.4(-0.3,9.1)$ & \\
\hline \multicolumn{7}{|l|}{$P$ for interaction $=0.499$} \\
\hline \multicolumn{7}{|l|}{ Any CAC progression ${ }^{\dagger}$} \\
\hline Diabetes, $n=148$ & $11.0(4.2,17.7)$ & $9.7(3.4,15.9)$ & $8.2(1.8,14.6)$ & $1.6(-8.6,11.8)$ & $0.4(-9.8,10.6)$ & $1.7(-8.5,11.9)$ \\
\hline
\end{tabular}


Model 1, unadjusted; Model 2, adjusted for age, sex and race; Model 3, adjusted for variables in model 2 plus BMI, current smoker status (yes/no), milliliter of daily alcohol consumption, SBP, antihypertensive medication use (yes/no), physical activity, serum creatinine, total cholesterol and LDL-C; Model 4, adjusted for variables in model 3 plus average of FG and change in FG level during variability measurement; Model 5 for individuals with diabetes, adjusted for variables in model 4 plus diabetes medication use before CAC assessment at year 25; Model 5 for individuals without diabetes, adjusted for variables in model 4 plus FG level at year 25. Model 6, for individuals with any CAC progression, adjusted for variables in model 4 plus baseline CAC. *Evaluated only among individuals without baseline CAC at the year 15 examination. ${ }^{\dagger}$ CAC progression was calculated as the difference of logarithmic CAC score at follow-up and baseline (log [CAC (follow-up) +1$]-\log [\mathrm{CAC}$ (baseline) +1$]$. 
Supplementary Table S7. Multivariable association between fasting glucose variation during study participants with CAC progression over 10 years (2000-2010) stratified by sex category

\begin{tabular}{|c|c|c|c|c|c|c|}
\hline & Model 1 & Model 2 & Model 3 & Model 4 & Model 5 & Model 6 \\
\hline \multicolumn{7}{|c|}{ \% CAC progression per 1-SD increase in FG-CV (95\% CI) } \\
\hline \multicolumn{7}{|l|}{ Incident $\mathrm{CAC}^{*}$} \\
\hline Male, $n=860$ & $9.8(4.9,14.7)$ & $10.8(6.0,15.7)$ & $8.3(3.4,13.1)$ & $3.5(-6.2,13.2)$ & $1.9(-7.9,11.7)$ & \\
\hline Female, $n=1,202$ & $8.1(5.0,11.2)$ & $7.6(4.5,10.8)$ & $5.2(2.1,8.3)$ & $9.5(6.6,12.5)$ & $8.9(5.9,11.9)$ & \\
\hline \multicolumn{7}{|c|}{$P$ for interaction $=0.539$} \\
\hline \multicolumn{7}{|c|}{ Any CAC progression ${ }^{\dagger}$} \\
\hline Male, $n=998$ & $8.3(3.9,12.7)$ & $9.0(4.7,13.3)$ & $6.7(2.3,11.0)$ & $3.2(-4.7,11.1)$ & $1.8(-6.4,10.0)$ & $3.6(-4.3,11.5)$ \\
\hline Female, $n=1,258$ & $10.1(7.1,13.0)$ & $9.7(6.7,12.7)$ & $6.8(3.8,9.7)$ & $11.0(8.3,13.8)$ & $10.8(6.6,15.1)$ & $10.4(5.0,15.7)$ \\
\hline \multicolumn{7}{|c|}{$\mathrm{P}$ for interaction $=0.499$} \\
\hline \multicolumn{7}{|c|}{$\%$ CAC progression per $1-S D$ increase in FG-SD $(95 \% \mathrm{CI})$} \\
\hline \multicolumn{7}{|l|}{ Incident $\mathrm{CAC}^{*}$} \\
\hline Male, $n=860$ & $11.4(6.5,16.3)$ & $12.2(7.4,17.0)$ & $9.4(4.8,14.1)$ & $1.9(-6.7,10.4)$ & $-0.3(-8.0,7.5)$ & \\
\hline Female, $n=1,202$ & $9.5(6.3,12.8)$ & $9.1(5.8,12.4)$ & $6.5(3.1,9.9)$ & $13.5(9.8,17.3)$ & $12.8(9.1,16.6)$ & \\
\hline \multicolumn{7}{|c|}{$P$ for interaction $=0.508$} \\
\hline \multicolumn{7}{|c|}{ Any CAC progression ${ }^{\dagger}$} \\
\hline Male, $n=998$ & $9.5(5.3,13.6)$ & $9.9(5.9,14.0)$ & $7.3(6.0,8.7)$ & $2.5(-4.1,9.1)$ & $1.2(-5.4,7.8)$ & $2.6(-4.0,9.1)$ \\
\hline Female, $n=1,258$ & $11.1(8.0,14.2)$ & $10.7(7.6,13.9)$ & $7.6(4.6,10.7)$ & $14.3(10.7,17.9)$ & $13.9(10.3,17.5)$ & $13.3(9.7,16.9)$ \\
\hline \multicolumn{7}{|c|}{$P$ for interaction $=0.518$} \\
\hline \multicolumn{7}{|c|}{ \% CAC progression per 1-SD increase in FG-ARV (95\% CI) } \\
\hline \multicolumn{7}{|l|}{ Incident $\mathrm{CAC}^{*}$} \\
\hline Male, $n=860$ & $11.8(6.5,17.1)$ & $12.7(7.5,18.0)$ & $9.8(4.7,14.9)$ & $1.5(-7.9,10.8)$ & $0.1(-2.6,2.7)$ & \\
\hline Female, $n=1,202$ & $9.9(6.8,12.9)$ & $9.5(6.5,12.6)$ & $7.3(5.0,9.7)$ & $8.8(6.4,11.2)$ & $8.3(5.9,10.8)$ & \\
\hline \multicolumn{7}{|c|}{$P$ for interaction $=0.509$} \\
\hline \multicolumn{7}{|c|}{ Any CAC progression ${ }^{\dagger}$} \\
\hline Male, $\mathrm{n}=998$ & $10.1(5.6,14.6)$ & $10.6(6.2,15.0)$ & $7.7(5.5,10.0)$ & $1.6(-2.7,5.8)$ & $0.7(-3.6,5.0)$ & $1.6(-2.7,5.8)$ \\
\hline
\end{tabular}


Female, $n=1,258$

Model 1, unadjusted; Model 2, adjusted for age and race; Model 3, adjusted for variables in model 2 plus BMI, current smoker status (yes/no), milliliter of daily alcohol consumption, SBP, antihypertensive medication use (yes/no), physical activity, serum creatinine, total cholesterol and LDL-C; Model 4, adjusted for variables in model 3 plus average of FG and change in FG level during variability measurement; Model 5, adjusted for variables in model 4 plus incidence of diabetes and diabetes medication use;. Model 6, for individuals with any CAC progression, adjusted for variables in model 4 plus baseline CAC. *Evaluated only among individuals without baseline CAC at the year 15 examination. ${ }^{\dagger} \mathrm{CAC}$ progression was calculated as the difference of logarithmic CAC score at follow-up and baseline (log [CAC (follow-up) +1$]-\log [\mathrm{CAC}$ (baseline) +1$])$. 
Supplementary Table S8. Multivariable association between fasting glucose variation during study participants with CAC progression over 10 years (2000-2010) stratified by race category

\begin{tabular}{|c|c|c|c|c|c|c|}
\hline & Model 1 & Model 2 & Model 3 & Model 4 & Model 5 & Model 6 \\
\hline \multicolumn{7}{|c|}{ \% CAC progression per 1-SD increase in FG-CV (95\% CI) } \\
\hline \multicolumn{7}{|l|}{ Incident $\mathrm{CAC}^{*}$} \\
\hline Black, $n=914$ & $8.3(4.8,11.7)$ & $7.8(4.4,11.2)$ & $5.9(2.4,9.4)$ & $4.0(-2.2,10.3)$ & $3.6(-2.8,10.0)$ & \\
\hline White, $\mathrm{n}=1,148$ & $12.9(8.1,17.6)$ & $11.2(6.6,15.7)$ & $9.7(5.2,14.2)$ & $6.4(-3.2,16.0)$ & $5.9(-3.7,15.5)$ & \\
\hline \multicolumn{7}{|c|}{$P$ for interaction $=0.122$} \\
\hline \multicolumn{7}{|c|}{ Any CAC progression ${ }^{\dagger}$} \\
\hline Black, $n=975$ & $8.5(5.1,11.8)$ & $8.0(4.8,11.3)$ & $6.3(2.9,9.6)$ & $3.8(-2.4,10.0)$ & $3.2(-3.0,9.5)$ & $4.7(-1.5,10.9)$ \\
\hline White, $n=1,281$ & $12.9(8.7,17.1)$ & $11.3(7.3,15.3)$ & $9.1(5.4,12.7)$ & $9.8(2.3,17.3)$ & $8.8(2.6,15.0)$ & $9.3(1.7,16.8)$ \\
\hline \multicolumn{7}{|c|}{$\mathrm{P}$ for interaction $=0.1$} \\
\hline \multicolumn{7}{|c|}{$\%$ CAC progression per $1-S D$ increase in FG-SD $(95 \% \mathrm{CI})$} \\
\hline \multicolumn{7}{|l|}{ Incident $\mathrm{CAC}^{*}$} \\
\hline Black, $n=914$ & $9.3(5.9,12.7)$ & $8.9(5.5,12.2)$ & $7.5(4.0,10.9)$ & $5.6(-2.5,13.6)$ & $5.2(-2.9,13.3)$ & \\
\hline White, $\mathrm{n}=1,148$ & $16.9(11.6,22.2)$ & $14.2(9.2,19.3)$ & $12.1(7.0,17.2)$ & $8.5(-7.3,13.8)$ & $8.7(-7.0,24.3)$ & \\
\hline \multicolumn{7}{|c|}{$P$ for interaction $=0.017$} \\
\hline \multicolumn{7}{|c|}{ Any CAC progression ${ }^{\dagger}$} \\
\hline Black, $n=975$ & $9.5(6.2,12.7)$ & $9.1(5.9,12.3)$ & $7.7(4.4,10.9)$ & $5.3(-2.7,13.3)$ & $4.8(-3.3,12.8)$ & $6.1(-1.8,14.1)$ \\
\hline White, $\mathrm{n}=1,281$ & $14.7(10.4,18.9)$ & $12.3(8.2,16.4)$ & $9.6(5.3,14.0)$ & $13.2(7.0,19.3)$ & $12.7(6.6,18.9)$ & $11.5(5.3,17.6)$ \\
\hline \multicolumn{7}{|c|}{$P$ for interaction $=0.048$} \\
\hline \multicolumn{7}{|c|}{ \% CAC progression per 1-SD increase in FG-ARV $(95 \% \mathrm{CI})$} \\
\hline \multicolumn{7}{|l|}{ Incident $\mathrm{CAC}^{*}$} \\
\hline Black, n=914 & $9.5(6.2,12.8)$ & $9.2(5.9,12.4)$ & $7.7(4.4,11.0)$ & $3.6(-1.6,8.7)$ & $3.4(-1.9,8.6)$ & \\
\hline White, $\mathrm{n}=1,148$ & $17.9(12.3,23.5)$ & $15.3(9.9,20.7)$ & $13.1(7.6,18.6)$ & $6.7(-3.5,16.9)$ & $6.8(-3.4,17.0)$ & \\
\hline \multicolumn{7}{|c|}{$P$ for interaction $=0.011$} \\
\hline \multicolumn{7}{|c|}{ Any CAC progression ${ }^{\dagger}$} \\
\hline Black, $n=975$ & $9.6(6.4,12.8)$ & $9.4(6.2,12.5)$ & $7.9(4.7,11.1)$ & $3.4(-1.7,8.5)$ & $3.1(-2.1,8.2)$ & $4.0(-1.1,9.1)$ \\
\hline
\end{tabular}


Model 1, unadjusted; Model 2, adjusted for age and sex; Model 3, adjusted for variables in model 2 plus BMI, current smoker status (yes/no), milliliter of daily alcohol consumption, SBP, antihypertensive medication use (yes/no), physical activity, serum creatinine, total cholesterol and LDL-C; Model 4, adjusted for variables in model 3 plus average of FG and change in FG level during variability measurement; Model 5, adjusted for variables in model 4 plus incidence of diabetes and diabetes medication use;. Model 6, for individuals with any CAC progression, adjusted for variables in model 4 plus baseline CAC. *Evaluated only among individuals without baseline CAC at the year 15 examination. ${ }^{\dagger} \mathrm{CAC}$ progression was calculated as the difference of logarithmic CAC score at follow-up and baseline (log [CAC (follow-up) +1$]-\log [\mathrm{CAC}$ (baseline) +1$])$. 
Supplementary Figure S1. Study flow chart. CARDIA, Coronary Artery Risk Development Study in Young Adults; CAC, coronary artery calcification.

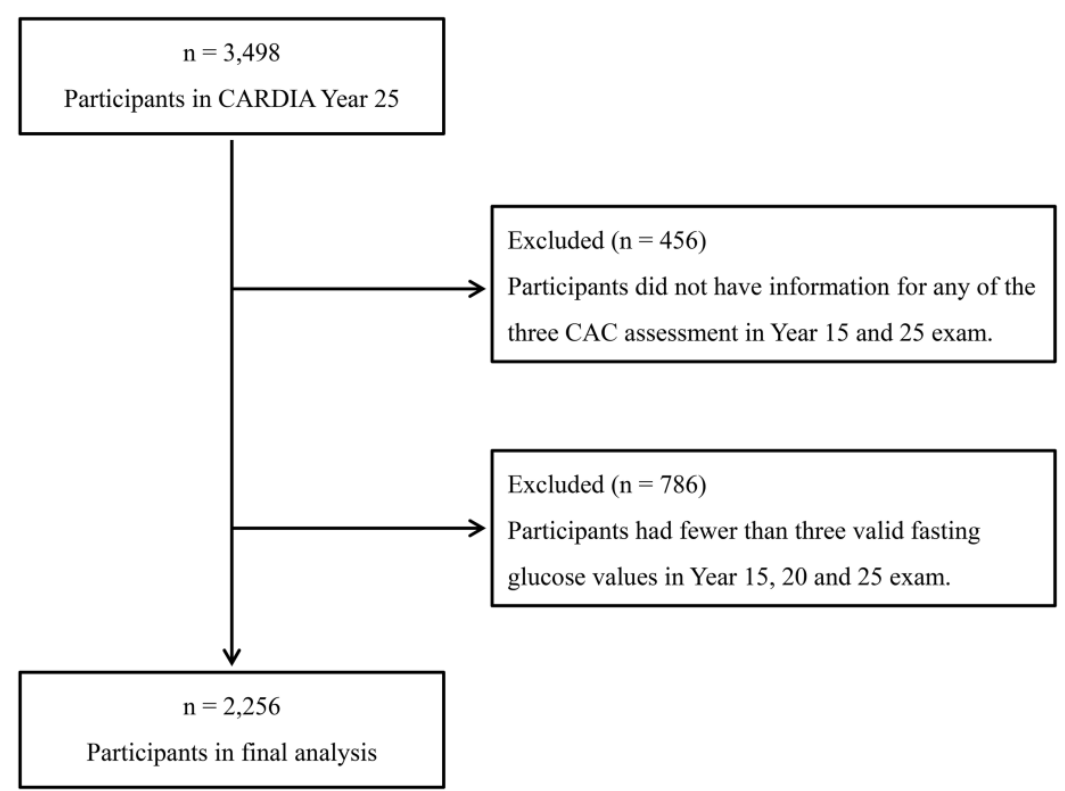


Supplementary Figure S2. Percentage of participants with CAC progression during 10-year follow-up period stratified by baseline CAC presence in the CARDIA study. CAC, coronary artery calcification.
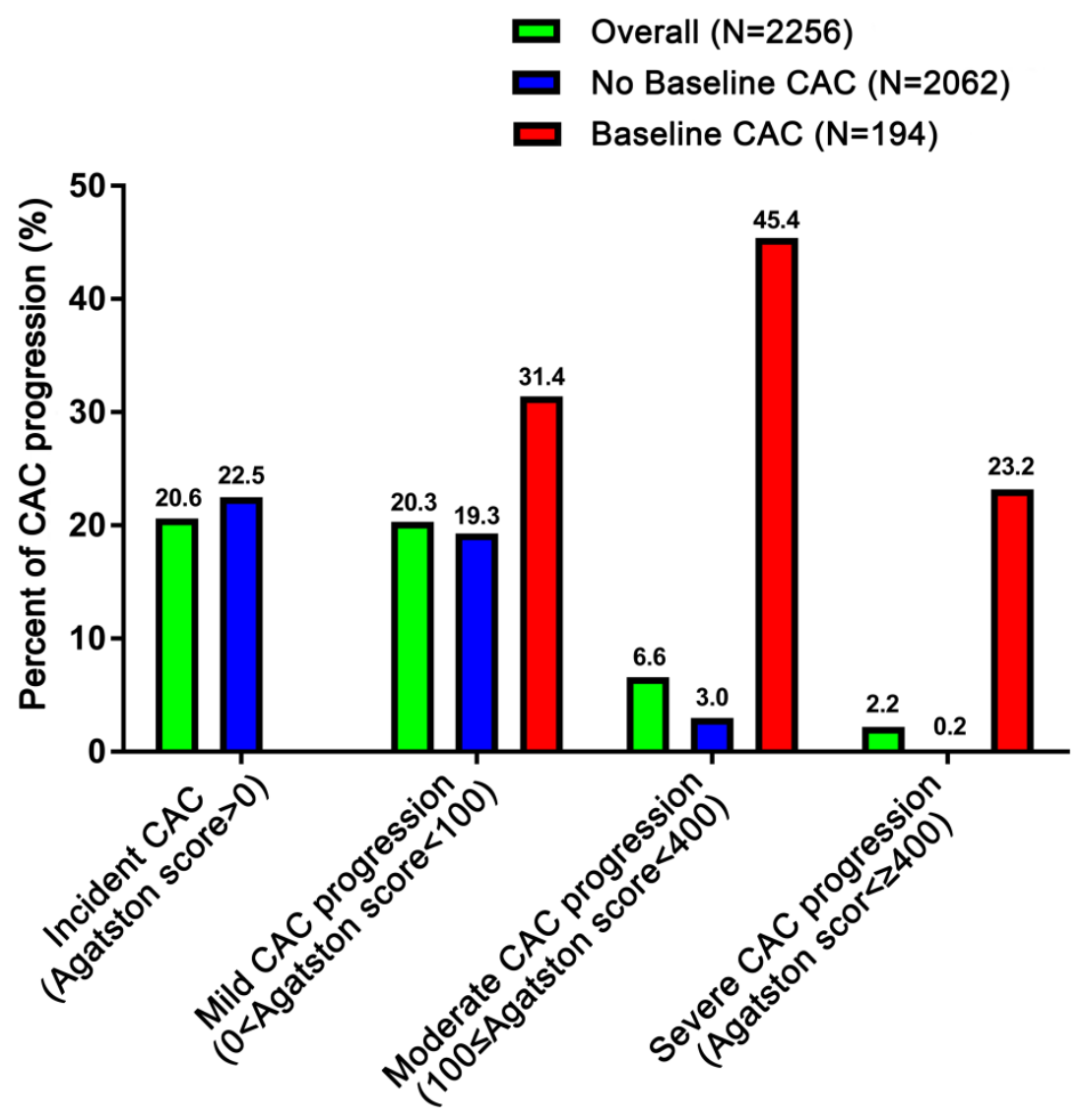
Supplementary Figure S3. CAC progression according to the quartile of fasting glucose variability. CAC score at baseline (Y15), 5-year (Y20) and 10-year (Y25) follow-up period stratified by the quartiles of (A) FG-SD, and (C) FG-ARV. CAC progression was gradually increased with quartiles of (B) FG-SD, and (D) FG-ARV during 10-year follow-up. ARV, average real variability; CAC, coronary artery calcification; FG, fasting glucose; SD, standard deviation.
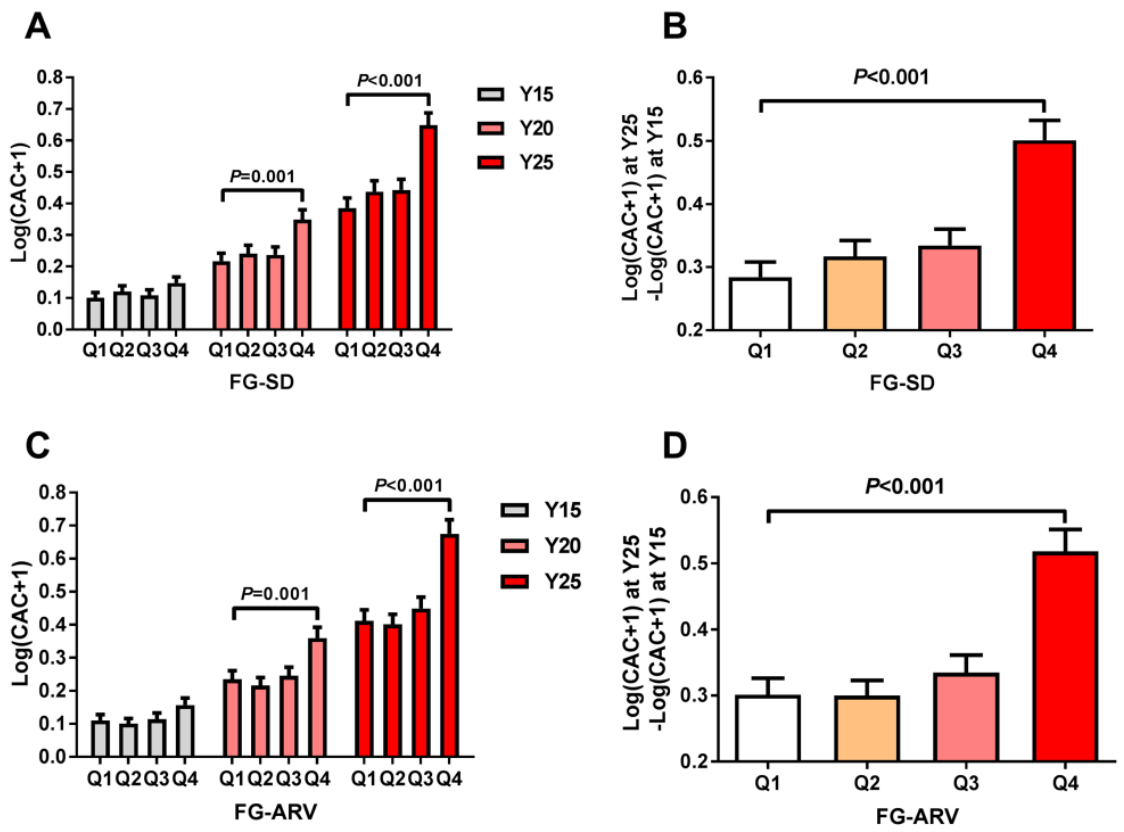


\section{Supplementary Figure S4.}

Forest plot of the association between a 1-standard deviation unit increment in fasting glucose coefficient of variation and percent progression of (A) incident CAC and (B) any CAC progression among individuals. Model adjustment: Demographics: age, sex and race; Cumulative Risk Factors: Demographics plus BMI, current smoker status, milliliter of daily alcohol consumption, SBP, antihypertensive medication use, physical activity, serum creatinine, total cholesterol and LDL-C; Average FG \& FG change: Cumulative Risk Factors plus average FG level and change in FG level during variability measurement; T2DM incidence \& Medication: Average FG \& FG change plus incidence of diabetes and diabetes medication use; Y25 FG level: Average FG \& FG change plus FG level at year 25; Baseline CAC: Average FG \& FG change plus baseline CAC.
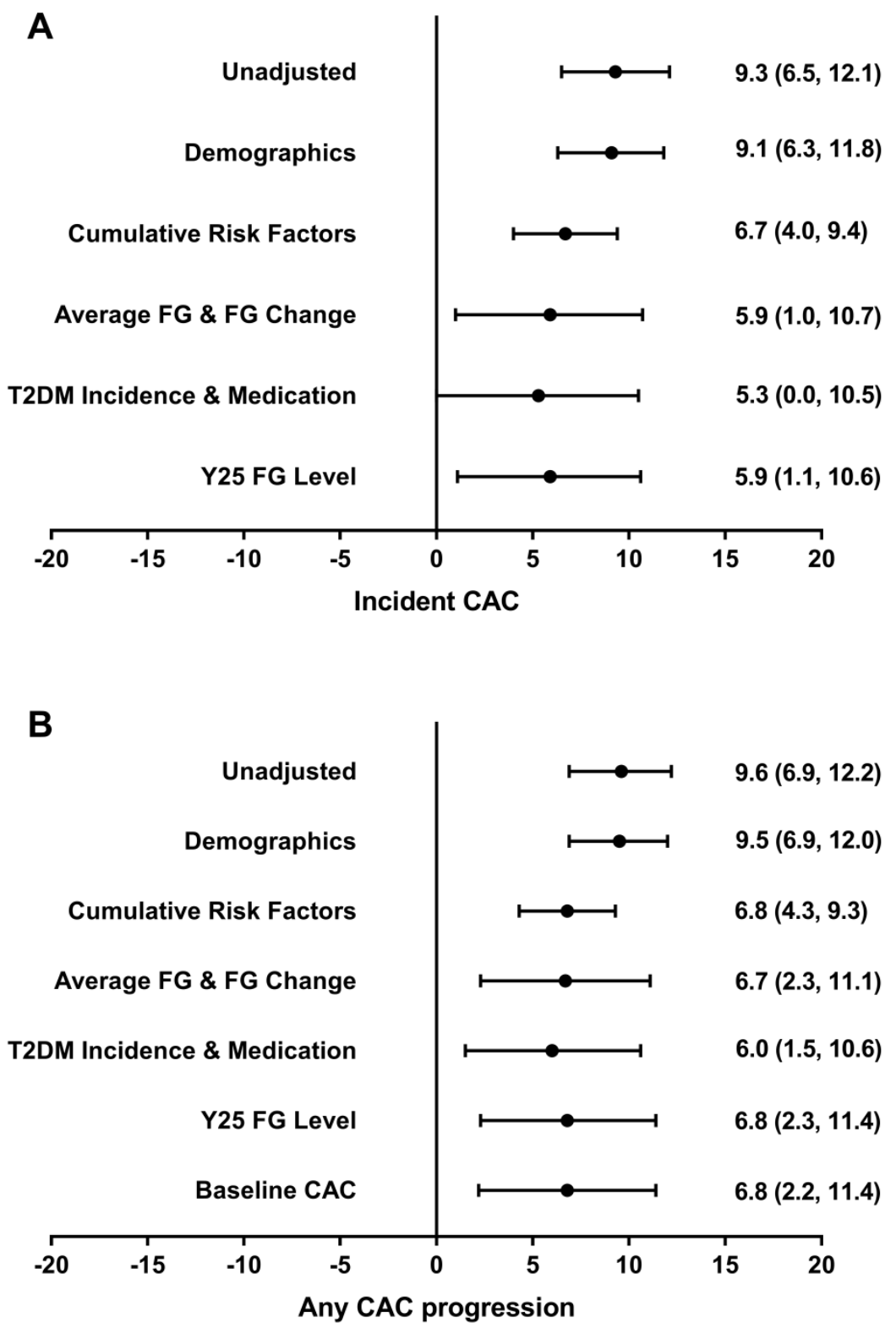
Supplementary Figure S5. Forest plot of the association between a 1-standard deviation unit increment in FG-SD and percent progression of incident CAC among individuals (A) with diabetes or (B) without diabetes and any CAC progression among individuals (C) with diabetes or (D) without diabetes. Model adjustment: Demographics: age, sex and race; Cumulative Risk Factors: Demographics plus BMI, current smoker status, milliliter of daily alcohol consumption, SBP, antihypertensive medication use, physical activity, serum creatinine, total cholesterol and LDL-C; Average FG \& FG change: Cumulative Risk Factors plus average FG level and change in FG level during variability measurement; T2DM Medication use: Average FG \& FG change plus diabetes medication use; Y25 FG level: Average FG \& FG change plus FG level at year 25; Baseline CAC: Average FG \& FG change plus baseline CAC. 
A

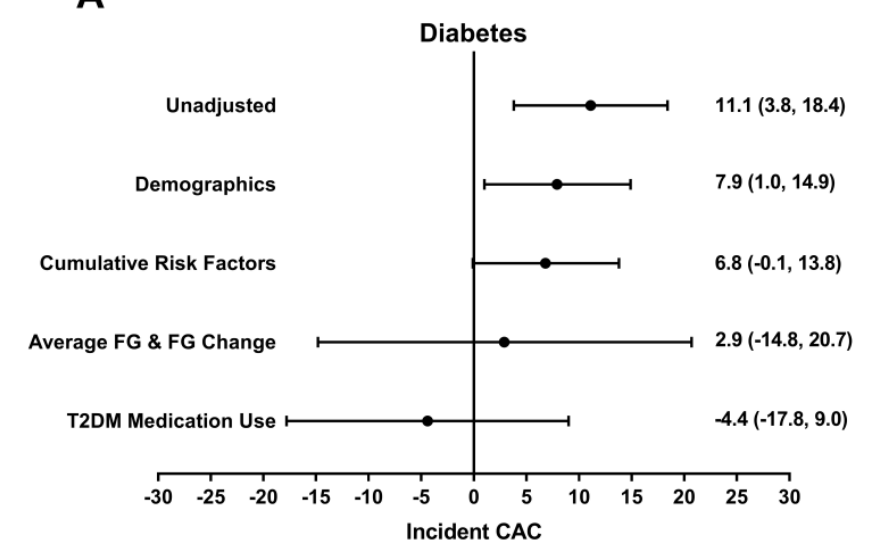

C

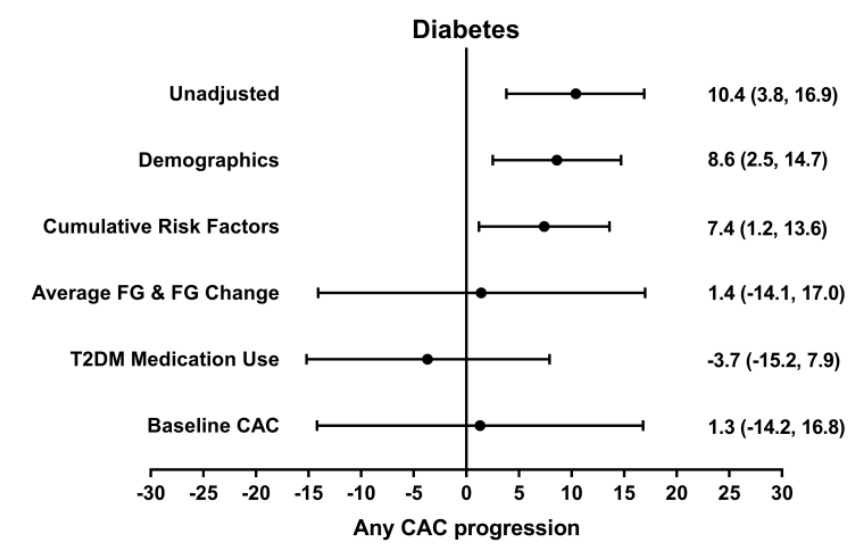

B

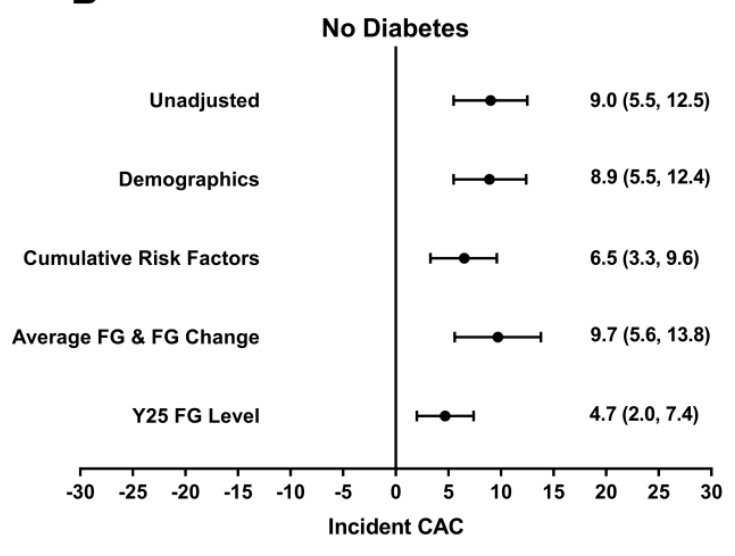

D

\begin{tabular}{|c|c|c|c|}
\hline \multicolumn{4}{|c|}{ No Diabetes } \\
\hline Unadjusted & & $\longmapsto$ & $9.5(6.4,12.7)$ \\
\hline Demographics & & $\longmapsto$ & $9.5(6.4,12.5)$ \\
\hline Cumulative Risk Factors & & $\longmapsto$ & $6.8(3.8,9.8)$ \\
\hline Average FG \& FG Change & & $\longmapsto$ & $11.6(7.7,15.4)$ \\
\hline Y25 FG Leve & & $\longmapsto$ & $5.6(1.0,10.2)$ \\
\hline Baseline CAC & & $\longmapsto$ & $11.0(7.2,14.8)$ \\
\hline $\begin{array}{cccc} & 1 & 1 & 1 \\
-30 & -25 & -20 & -15\end{array}$ & $\begin{array}{ll}10 & -5 \\
-10 & -5\end{array}$ & $\begin{array}{lll}15 & 10 & 15\end{array}$ & $\begin{array}{lll}10 & 25 & 30\end{array}$ \\
\hline
\end{tabular}


Supplementary Figure S6. Forest plot of the association between a 1-standard deviation unit increment in FG-ARV and percent progression of incident CAC among individuals (A) with diabetes or (B) without diabetes and any CAC progression among individuals (C) with diabetes or (D) without diabetes. Model adjustment: Demographics: age, sex and race; Cumulative Risk Factors: Demographics plus BMI, current smoker status, milliliter of daily alcohol consumption, SBP, antihypertensive medication use, physical activity, serum creatinine, total cholesterol and LDL-C; Average FG \& FG change: Cumulative Risk Factors plus average FG level and change in FG level during variability measurement; T2DM Medication use: Average FG \& FG change plus diabetes medication use; Y25 FG level: Average FG \& FG change plus FG level at year 25; Baseline CAC: Average FG \& FG change plus baseline CAC. 
A

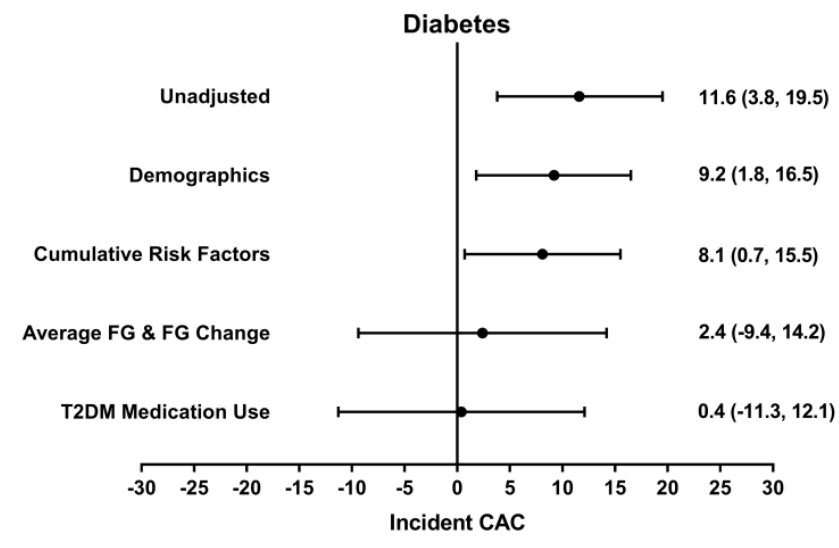

C

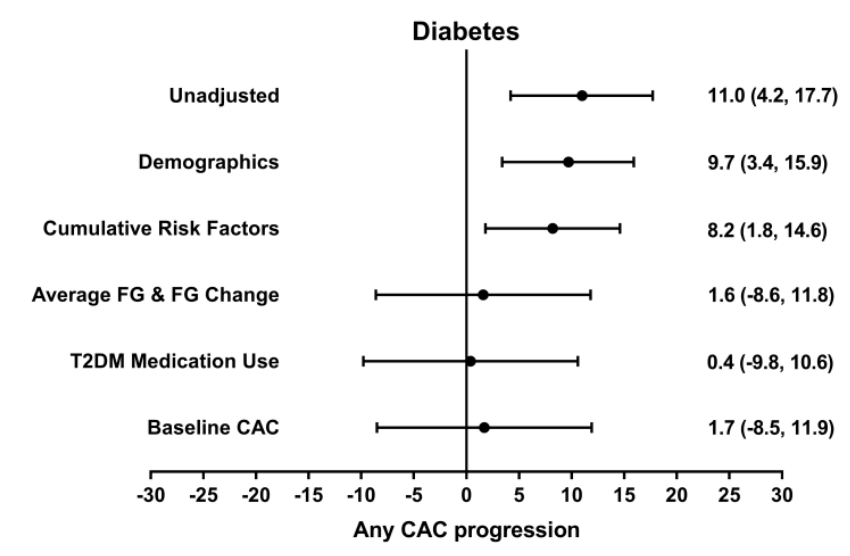

B

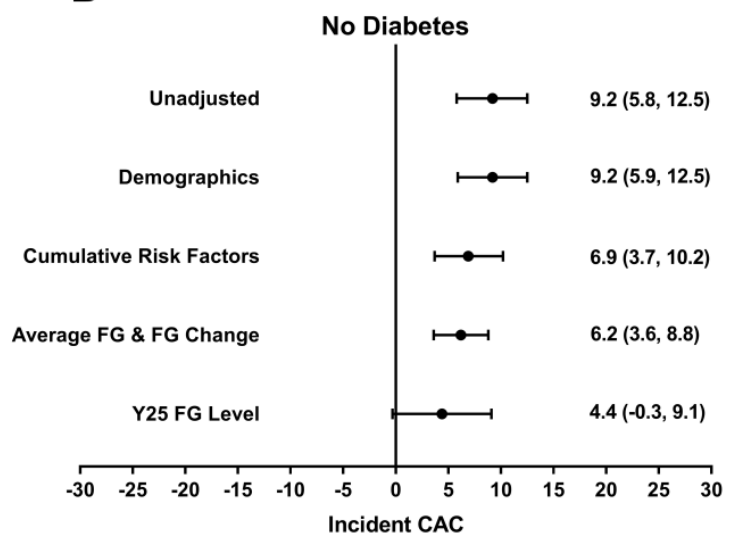

D

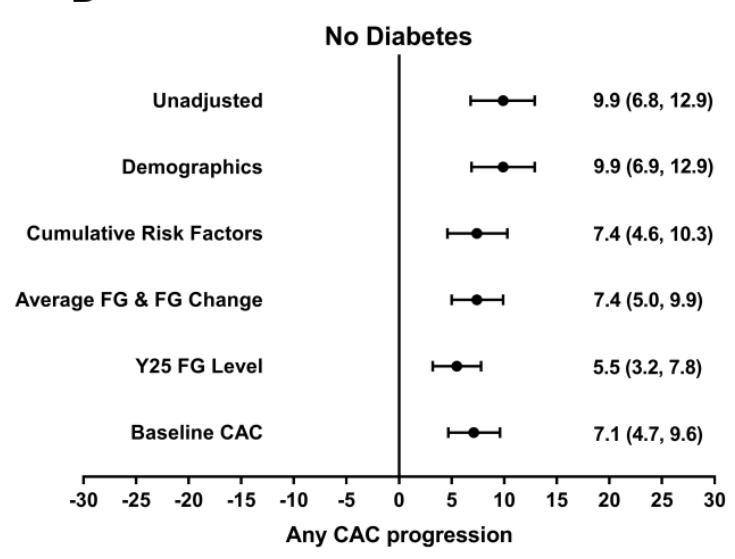

